\title{
Towards a Coherent Theory of Physics and Mathematics: The Theory-Experiment Connection
}

\author{
Paul Benioff*
}

\begin{abstract}
The problem of how mathematics and physics are related at a foundational level is of much interest. One approach is to work towards a coherent theory of physics and mathematics together. Here steps are taken in this direction by first examining the theory experiment connection. The role of an implied theory hierarchy and use of computers in comparing theory and experiment is described. The main idea of the paper is to tighten the theory experiment connection by bringing physical theories, as mathematical structures over $C$, the complex numbers, closer to what is actually done in experimental measurements and computations. The method replaces $C$ by $C_{n}$ which is the set of pairs, $R_{n}, I_{n}$, of $n$ figure rational numbers in some basis. The properties of these numbers are based on the type of numbers that represent measurement outcomes for continuous variables.

A model of space and time based on $R_{n}$ is discussed. The model is scale invariant with regions of constant step size interrupted by exponential jumps. A method of taking the limit $n \rightarrow \infty$ to obtain locally flat continuum based space and time is outlined. Possibly the most interesting result is that $R_{n}$ based space is invariant under scale transformations which correspond to expansion and contraction of space relative to a flat background. Also the location of the origin, which is a space and time singularity, does not change under these transformations. Some properties of quantum mechanics, $Q m_{n}$ based on $C_{n}$ and on $R_{n}$ space are briefly investigated.
\end{abstract}

\footnotetext{
*Physics Division, Argonne National Lab, Argonne, IL 60439; e-mail: pbenioff@anl.gov
} 


\section{Introduction}

As is widely recognized, quantum mechanics and its generalizations, such as quantum field theory, is a highly successful theory. So far it has survived every experimental test. Yet in spite of this problems remain. The problem of measurement is one. Although the use of decoherence to solve the problem(1,2) helps in that it explains the existence of the pointer basis in measuring apparatuses, questions still remain ${ }^{(\underline{3})}$ that are related to whether quantum mechanics is really a theory of open systems only or whether there is a system such as the universe that can be considered to be closed and isolated. This is the approach taken by the Everett Wheeler interpretation (4.5) .

There are other open questions such as, why is space-time $3+1$ dimensional, why are there four fundamental forces with the observed strengths, what is the reason for the observed elementary particle mass spectrum, and why did the big bang occur. A recent list of ten basic questions $(\underline{\underline{6}})$ includes these and other questions. Another basic question relates to why quantum mechanics and its extensions is the correct physical theory.

There are papers in the literature that address some of these questions by attempting to show that if things were different then life could not have evolved or some physical catastrophe would happen $\underline{(7.8 .9 .10}$. However these are all heuristic after-the-fact types of arguments and do not constitute proofs. The possibility of constructing a theory to explain these things, as a "Theory of Everything" or TOE represents a sought after goal of physics (11.12.13.10).

Another very basic problem concerns the relation between physics and mathematics. The view that seems to be taken by most physicists is that the physical universe and the properties of physical systems exist independent of and a-priori to an observers use of experiments to construct a theory of the physical universe. In particular it is felt that the properties of physical systems are independent of the basic properties of how an observer acquires knowledge and constructs a physical theory of the universe. This view is expressed by such phrases as "discovering the properties of nature" and regarding physics as "a voyage of discovery".

A similar situation exists in mathematics. Most mathematicians appear to implicitly accept the realist view. Mathematical objects have an independent, a priori existence independent of an observers knowledge of them $\frac{(14.15)}{}$. Much mathematical activity consists of discovering properties of these objects.

This is perhaps the majority view, but it is not the only view. Other concepts of existence include the formalist approach and various constructive approaches $\underline{(16.17 .18 .19})$. These approaches will not be used here as they do not seem to take sufficient account of limitations imposed by physics, e. g. the physical nature of language $\underline{(20)}$ and information $\underline{(21)}$.

This realist view of physics and mathematics has some problems. This is especially the case if one considers physical systems as those that exist in and determine properties of a space-time framework and mathematical systems as those that exist outside of space-time and have nothing to do with space-time. If this is indeed the case, then why should mathematics be relevant or useful at all 
to physics? Also if this is the case, it is not clear how one acquires knowledge of mathematics $(15)$. It is obvious that physics and mathematics are closely entwined, as is shown by extensive use of mathematics in theoretical physics, yet it is not clear how the two are related at a foundational level.

This problem has been well known for a long time and has been much studied. An early and well known expression of it was by Wigner $\stackrel{(22)}{2}$ in a paper in 1960 entitled The Unreasonable Effectiveness of Mathematics in the Natural Sciences. A sampling of the extensive literature on various aspects of the relationship between physics and mathematics includes work on quantum set theory ${ }^{(23.24 .25 .26)}$, the relationship between the Riemann hypothesis and aspects of quantum mechanics $(27.28)$ and relativity $(\underline{29)}$, and efforts to connect quantum mechanics and quantum computing with logic, languages, and different aspects of physics ${ }^{(30.31)}$ along with efforts to connect mathematical logic with physics $\left({ }^{7.32 .33}\right)$. A related question that needs addressing is, Why is Physics so Comprehensible? ${ }^{(34)}$.

Other foundational issue are based on the universal applicability of quantum mechanics. If quantum mechanics is truly universally applicable, then all physical systems, including experimental equipment, computers, and even intelligent systems and language expressions are quantum systems in different states. The fact that intelligent systems, and experimental equipment are macroscopic and follow a dynamics that, for most or all variables of interest, is classical mechanical does not exclude their following quantum dynamical laws. Examples of such laws are evolution equations for density operators which are interacting with their environment.

An important example of dynamical processes is a process by which an intelligent system compares theoretical predictions with experimental outcomes as part of the process of validation or refutation of a theory. If quantum mechanics is universally applicable, then both experimental apparatus and computers are quantum dynamical systems as is the intelligent system that is carrying out the validation. In addition the expressions in the language of the theory from which the theoretical predictions are obtained are also states of quantum systemst ${ }^{20}$.

It follows that the process of validation (or refutation) of any theory is a quantum dynamical process described by quantum dynamical evolution laws. If these ideas are applied to quantum mechanics itself, then one sees that quantum mechanics must in some sense describe its own validation by quantum systems. However almost nothing is known so far about the details of such a description. A possible model that may be useful for studying this problem is a quantum robott ${ }^{35}$ as a mobile quantum computer interacting with its environment and generating output binary qubit states of increasing length. One can ask if there exists a quantum dynamics for the robot such that the output binary strings it generates can be interpreted by us, as external observers, as language expressions that have meaning to us. Can these expressions have meaning as descriptions of experimental tests of predicted properties of the robots environment? Perhaps more importantly one can ask if the binary states have meaning to the quantum robot itself, and if so, what the meaning is.

The approach taken here is to work towards the development of a coherent 
theory of physics and mathematics that describes both physical and mathematical systems together in a single theory rather than as two separate types of systems with different theoretical descriptions. It is hoped that such a theory, with its somewhat different view of physics and mathematics, may help in answering some of the open questions described above.

The next section provides a description of some aspects of a coherent theory of physics and mathematics. The description is brief because it is based on previous descriptions of this approacht $20,36.37^{\circ}$. It is presented here because it provides an overarching foundation or basis in which to frame and investigate questions of interest in this paper. However it and subsections 3.1 and 3.2 can be skipped by readers interested mainly in the physical aspects discussed in this work.

The main part of this paper is contained in Sections 3 - 6 Various aspects of the theory experiment connection that are of interest here are discussed in Section 3 Included is a discussion of the indirectness of the status of physical reality as a function of the size of a system. It is seen that if one takes into account the requirements that equipment for an experiment functions properly and that this depends on the validity of theories on which the proper functioning is based, then a hierarchy of theories or parts of theories results. The question of why computers are necessary to compare theory with experiment is discussed from a slightly different viewpoint than that usually assumed. In essence the point is that computers provide a bridge between the type of real number names given by theory and the type of number generated as experimental output.

The next two subsections discuss this connection, but more from the viewpoint of regarding real numbers as elements of equivalence classes of convergent Cauchy sequences of rational string numbers ${ }^{1}$, and measurement outcomes as finite digit strings in some basis, i.e. an $n$ significant figure representation. The use of coarse graining to connect the two types of numbers is noted along with some problems with this connection.

The main point of the paper is in subsection 3.4. There it is proposed that the theory-experiment connection be tightened up. The method suggested is based on the observation that all physical theories to date can be described as different mathematical structures over $C$, the field of complex numbers. The method consists in replacing $C$ by $C_{n}$ which is a set of finite string complex rational numbers of length $n$ in some basis (e.g. binary) and then taking the limit $n \rightarrow \infty$. In this way one starts with physical theories based on numbers that are much closer to experimental outcomes and computational numbers than are $C$ based theories.

The rest of the paper describes some consequences of this replacement. Most

\footnotetext{
${ }^{1}$ One may also regard real numbers as infinite digit strings in some basis. However this requires including in a theory language expressions of infinite length. This is excluded as language expressions are required to be of finite length. Rational number strings are finite digit strings with digits different from 0 in both sides of the point separating positive and negative powers of the basis, as in 1101.011 in binary. Even though this does not include all rational numbers, rational number strings are dense in the rational numbers. As a result, each real number corresponds to a convergent Cauchy sequence of these string numbers.
} 
of the discussion, in Section 4 centers on some aspects of $R_{n}$ based space and time. The description of space and time, which is based on the properties of numbers in $R_{n}$, the real part of $C_{n}$, is very different from the usual description. In essence it is a scale invariant description in that multiplication of the space time locations by any power of the base used in $R_{n}$ does not change anything. Interesting results include the nature of the origin as a singularity and the observation that scale transformations correspond to expansion and contraction of the space relative to a flat background without changing the location of the origin.

The next two Sections, 5 and 6 give brief descriptions of a few aspects of $Q m_{n}$, which is $C_{n}$ based quantum mechanics, and of taking the limit $n \rightarrow \infty$. The limit process is quite important in that one must recover continuum based space and time and $C$ based physics in the limit. The paper concludes with a summary in Section 7

The work described here represents initial steps in approaching a coherent theory of physics and mathematics. One may hope that these or related foundational questions and the approach taken here are also of interest to Asher Peres. His interest in foundational aspects regarding the interpretation of quantum mechanicst $38^{\circ}$ do overlap those of this paper. It is a pleasure to submit this paper as part of a 70th birthday festschrift for Asher Peres.

\section{Towards a Coherent Theory of Physics and Mathematics}

As noted above the goal of a coherent theory of physics and mathematics is to treat mathematical and physical systems together in a single unified theory rather than regarding them as completely distinct types of systems described by different theories. At present essentially nothing is known about the details of a coherent theory. However one can summarize some basic properties that a coherent theory should have ${ }^{36}$.

Probably the most important property is that it mathematics and physics are treated together in a single coherent theory instead of treating them as separate entities as is presently done. At the outset one should set out what a theory is in general. The position taken here is based on the description provided by mathematical logic which is the study of theories in general.

Mathematical logic makes a clear separation between formal theories and a universe of objects which the theory is supposed to describe. A formal theory $T$ is based on a language $\mathcal{L}(T)$ of expressions as strings of symbols. Expressions in $\mathcal{L}(T)$ are characterized in the usual wayl ${ }^{39}$ ås terms built up from function, variable, and constant symbols and as predicates built up from predicate symbols and logical connectives. A theory $T$ is created from the language by a choice of a set of formulas (i. e. predicates) as the axioms of the theory ${ }^{2}$. The axioms,

\footnotetext{
${ }^{2}$ The set of axioms is required to be decidable $39.40^{2}$. This is done to avoid choices of undecidable sets as axioms such as the set of all true statements for some interpretation as
} 
along with the logical axioms and logical rules of deduction, which are common to all theories, are used to define proofs as strings of formulas. A theorem is a terminal formula of a proof and a formula is a theorem if and only if it has a proof.

It will be assumed here that a coherent theory is axiomatizable using first order predicate logic. Whether this is the case or not is not known at present. The usefulness of this assumption lies in the availability of a large body of knowledge for first order theories. Also all the mathematics used so far by physics, and almost all mathematics, is covered by first order theories. ${ }^{3}$

The universe that the theory is supposed to describe consists of physical and mathematical systems. Hopefully it includes at least most or all of the physical universe and as much of mathematics as is needed. Also it is not known how such a theory will distinguish between physical and mathematical systems, if such a distinction does exist. However the present situation, viewing mathematical objects as independent of and outside space time and physical objects as inside of and determining the properties of space time, is not tenable.

It should be emphasized that readers may disagree with the description of mathematical and physical universes presented here. Different positions include belief in the existence of many universes ${ }^{44.4 .5}$, and the belief that mathematical existence is different from the Platonic view. (For a recent review see Marek and Mycielski1 ${ }^{45}$. From the viewpoint of this paper, it does not matter what the readers specific beliefs are about the ontology of physics and mathematics. It is sufficient to start from the position that mathematical and physical systems are different and distinct and have a different ontological status.

The connection between a formal theory and its language, and a mathematical system or structure consisting of individuals, functions, and relations or properties is through an interpretation, $I$, or map from the symbols, terms, and formulas of the language to the mathematical system. Variable and constant symbols are mapped to variables and constants in the system. Terms are mapped to terms and formulas are mapped to relations in a straightforward manner that mirrors the inductive description of expressions in $\mathcal{L}(T)$ that are terms and formulas. The mathematical structure for $T$ and $\mathcal{L}(T)$ is a model of $T$ and $\mathcal{L}(T)$ if and only if all axioms of $T$ are true in the structure. This assumes that $T$ is consistent (not all formulas are theorems). Otherwise $T$ has no modelst ${ }^{39.41}$. Note that the syntactic concept of provability or theoremhood as a property of formulas in $T$ is quite separate from the semantic property of true or false that applies to relations in the mathematical structure.

For any physical theory there is also the requirement that it be validated by agreement between theory and experiment. This extra connection, which is not part of mathematical theories, is essential. It is also an essential connection for a coherent theory of physics and mathematics. If one regards a physical theory as a formal theory with an interpretation into some mathematical structure

\footnotetext{
the axioms.

${ }^{3}$ Second order logic quantifies over both individuals, as is done in first order logic, and over sets of individualst 43 . One hopes that the increased strength of second order logic is not needed here.
} 
then a physical theory can be consistent and thus valid mathematically. This has nothing to do with whether it is valid or invalid physically (i.e. agrees or disagrees with experiment).

This situation is unsatisfactory in that one would like to bring these two notions of validity together in some fashion. It is hoped that a coherent theory of physics and mathematics together would provide some insight into how this might be accomplished. It is hoped that the work described here in the following sections about the theory experiment connection might help in this endeavor.

Another aspect of the relation between a formal physical theory $T$, the theory language $\mathcal{L}(T)$, and mathematical and physical validation of $T$ is based on the physical nature of all representations of language, i.e. "language is physical" $\mathfrak{l 0}^{20}$. Examples include written material, such as the text of this paper, speech, and trains of electromagnetic signals representing digitized text. Physically, printed text consists of ink molecules at different locations on a periodic space lattice and speech consists of amplitude modulations of a train of sound waves or of electromagnetic signals, such as transmission over telephone lines. Electromagnetic transmission of digitized text is an essential component of communication via the internet.

The existence of physical representations is essential. Without them it would not be possible to communicate anything or to even think about theories. Theoretical explanations of what was directly experienced would not be possible. Note that here the emphasis here is on the direct physical nature of all representations of a language, not on a theoretical description of the physical representations of the language. Such a description and its relation to the representation is of much interest, though, as it leads to questions regarding the possible existence of formulas that describe their own physicst ${ }^{20}$.

\section{The Theory-Experiment Connection}

In this section some aspects of the comparison between theoretical predictions and experiment will be discussed. Much of the discussion will apply to quantum mechanics. Most but not all of the points discussed here, although known implicitly, are usually not discussed explicitly. Also some novel suggestions about the theory experiment connection will be made.

\subsection{Immediacy of Reality as a Function of System Size}

An interesting aspect of the status of reality or objectivity for physical systems is that the directness or immediacy of the reality status of physical systems depends on the system sizel 36.37 . To see this one notes that the reality status, or existence, of very small physical systems or very large and far away systems, is more indirect than is the reality status of systems that are, or are perceived to be, moderate sized ( our size) systems. For instance "This rock is heavy, rough and white" are immediate directly observed properties of a laboratory sized system, a rock. The reality status of these properties is immediate and 
direct as they are direct sense impressions. No theory is needed to experience these properties. Another example is that "the sun is hot, round, and moves through the sky". These are directly experienced properties of a system whose perceived size is a few $\mathrm{cm}$.. No theory is needed for these properties; their reality status is immediate and direct.

The reality status of small systems such as bacteria, and even smaller systems such as atoms and molecules is more indirect in that it depends on the proper functioning of equipment used to observe these systems. The proper functioning of the equipment depends on theory supported by experiment that describes how the equipment operates and that it performs as expected. One concludes from this that the existence and other properties of systems, such as bacteria, atoms, and molecules, depends on intervening layers of theory supported by experiments. In this sense the reality status of these small systems is more indirect than that of rocks or of equipment used to observe the systems.

In this sense the reality status of smaller systems such as protons and neutrons and the elementary particles of physics is even more indirect as it depends on many layers of intervening theory supported by experiment that describe the proper functioning of even more complex equipment.

The same holds for large, far away cosmological systems. The existence and other properties of these systems depends on the proper functioning of telescopes and complex recording equipment. This in turn depends on all the theory supported by experiment needed to ensure that a telescope and much other associated equipment functions properly and is not just a meaningless assembly of parts.

The sun is a good example to show that some properties of a system are more indirect with more levels of intervening theory and experiment than for other properties. The properties of the sun noted above are immediate and direct. The large size and distance of the sun and the gravitational attraction between the sun and planets are less direct in that they depend on Euclidean geometry and the Newtonian theory of gravity and supporting experiments. Observing and understanding the spectra of light emitted from the sun is even more indirect in that it is based on the theory of electromagnetism and the quantum mechanics of atoms and molecules and supporting experiments that both validate these theories and determine the proper function of relevant experimental equipment. Finally the energy source of the sun as thermonuclear fusion is even more indirect as it depends on all the theories noted above and nuclear theory plus special relativity.

These examples and many others can be described more abstractly by noting that the validity of an experimental test of a theoretical prediction depends on the proper functioning of each piece of equipment used in the experiment. But the proper functioning of each piece of equipment depends in turn on other supporting theory and experiments which in turn.. . For example, suppose an experiment to test the validity of a theory uses two pieces of equipment, $E_{1}, E_{2}$. The validity of this experiment as a test depends on the proper functioning of $E_{1}$ and $E_{2}$. However, the proper functioning of $E_{1}$ also depends on some theory which may or may not be the same as the one being tested, and also on some 
other experiments each of which depend on other pieces of equipment for their validity. This argument then applies also to the experiments used to validate the theory on which the proper functioning of $E_{1}$ is based. Similar statements can be made for the proper functioning of $E_{2}$.

Basic examples of such equipment are those that measure time and distance. The truth of the assertion that a specific system, called a clock, measures time depends on the theory and experiments needed to describe the clock components and their proper functioning. The proper functioning of a clock depends on the proper functioning of each component of a clock. Similar arguments can be made for distance measuring equipment and equipment for measuring other physical parameters.

Computations made to compare theoretical predictions with experiment have the same property. A computation is a sequence of different steps each performed by a computer which is a physical system. The proper functioning of the computer depends on the proper functioning of the many parts of the computer. This is based on a theory, which may or may not be the same as the one for which the computation is made, and on supporting experiments that validate the theory on which the proper functioning of the computer and its parts is based. This dependence is also required to conclude that the outcome of a dynamical process that describes the operation of a computer is indeed a computation.

This shows that the reality status of system properties depends on a downward descending network of theories, computations, and experiments. The descent terminates at the level of the direct, elementary observations. These require no theory or experiment as they are uninterpreted and directly perceived.

The indirectness of the reality status of systems and their properties is measured crudely by the depth of descent between the property statement of interest and the direct elementary, uninterpreted observations of an observer. This can be described very crudely as the number of layers of theory and experiment between the statement of interest and elementary observations. The dependence on size arises because the descent depth, or number of intervening layers, is larger for very small and very large systems than it is for moderate sized systems.

The same arguments can be made for the complexity or indirectness of experimental support for a theory. The validity of each experiment as a test of a theory also depends on a downward descending network of theories, computations, and experiments that terminates at the level of direct, elementary observations. The theory being tested by the experiment may or may not be the same as the theory used to support the proper functioning of equipment used in the experiment.

Another measure of the indirectness of the reality of properties or indirectness of experimental support for a theory is the amount of physical resources needed to carry out the necessary experiments and computations. It is clear that the resources need to carry out experiments to determine properties of very small or very large far away systems are greater than those needed for moderate sized local systems. This approach will not be pursued here as it is 
discussed elsewheret ${ }^{37}$.

Finally it must be emphasized that the discussion is about the indirectness of the reality status or existence of systems and their properties only. It is not at all suggested or implied that some systems and properties are more or less real than others.

\subsection{Why Computers?}

At first sight the question of why computers are needed for theory experiment comparison has an obvious answer: To compute numbers for theoretical predictions to compare directly with the results of experiments. Here a slightly different viewpoint is taken that fits in with the material in Section 2 and with the possibility of a coherent theory of physics and mathematics.

To begin with one must consider the naming or designation of real numbers as these are the assumed connection between theory and experiment. That is, in quantum mechanics at least, all predictions of theory are real numbers. This follows from the basic mathematical structure of quantum mechanics. This is the case even for systems in discrete states such as spin projections. Here the eigenvalues of the Pauli operators such as \pm 1 for $\sigma_{z}$ are integers only through the inverse of an obvious map of integers to real numbers, $\pm 1 \mapsto \pm 1.0000 \cdots$.

The physical nature of language requires that real number names must be strings of symbols. One possibility that comes to mind is to extend any $k$-ary representation used for the rational numbers to include infinitely long symbol strings. If this were possible, then each real number would have a name. The problem is that such strings are excluded on physical grounds in that all symbol strings must be finite in length.

An immediate consequence of this requirement is that almost all real numbers cannot be named as there are only a countable infinity of finite symbol strings. Thus one needs to examine how names of real numbers can be named under this limitation.

In general there are several ways to name some real numbers. For a $k$-ary representation one type of name is $\underline{a} *(\underline{b})^{\infty}$. Here $\underline{a}$ and $\underline{b}$ denote finite sequences of $k$ digits and the superscript denotes infinite repetition of $\underline{b}$. The symbol $*$ denotes concatenation. The location of the " $k=a l "$ point, which is arbitrary, denotes the magnitude of the number.

As is well known, this type of naming gives names to the real number equivalents of all rational numbers. Another type of naming corresponds to replacing an infinite $k$-ary sequence by a computation procedure that can compute successive approximations to the infinite sequences. Such a procedure might, for example, compute for each $n$ the first $n$ binary digits of an infinite string. Since there are at most countably many computation procedures under any given representation of computability, e.g. as Turing machinest ${ }^{42}$, there are at most countably many computable real numbers. Here a real number is computable if there is a Turing machine that can compute successive approximations to the number. In this case, computable real numbers can be given a (natural number) 
name corresponding to the position, in a listing of all Turing machines, of any Turing machine that approximates the number.

This method of naming gives many names to each computable real number as there are many possible ways to compute successive approximations to a given number. It includes the real number representations of rational numbers as well as real numbers such as $e, \pi, \sqrt{2}$, and many others expressible as the limit of a computable convergent series.

Another method of naming real numbers is based ultimately on the property that each polynomial equation of odd degree has a real number solution. Algebraic real numbers are a special case of this in that the coefficients of the polynomials are integers. Since all integers (and rational numbers) have names, each algebraic real number $r$ can be named by a polynomial equation, $\sum_{j=0}^{n} a_{j} x^{j}=0$ where each $a_{j}$ is an integer, which has $r$ as a solution. If the equation has many solutions, then naming requires an additional specification, such as that based on use of an ordering (e.g. the nth smallest solution).

Physical theories greatly extend this method of naming real numbers in that all theoretical predictions are expressed as equations which supposedly have real number solutions. This is the case for any physical theory that describes a mathematical structure over a field of complex numbers. Quantum mechanics and its generalizations, such as quantum field theory, QED, and QCD are prime examples with this structure. So are special and general relativity. In these cases predictions correspond to theorems as statements in the theory language that define unique real numbers that are solutions of equations described in the theorem. Note that each coefficient appearing in these equations must be a name of some specific element representing a dimensional or dimensionless physical quantity in the mathematical structure of the theory.

To see this in more detail it is worth looking at a specific example in quantum mechanics. Suppose one is interested in the low lying atomic energy levels of an atom of mass $M$ with $Z$ electrons. The prediction of the ground state energy of the of the atom as the lowest eigenvalue of the Hamiltonian $\tilde{H}$ is obtained by solving the time independent Schrödinger equation $\tilde{H} \Psi-E \Psi=0$ for the energy of the ground state. The Hamiltonian has the well known form in the space coordinate representationl ${ }^{47}$,

$$
\tilde{H}=\sum_{j=1}^{Z}\left(-\frac{\hbar^{2}}{2 m} \underline{\nabla}_{j}^{2}\right)-\frac{\hbar^{2}}{2 M} \underline{\nabla}_{0}^{2}+\sum_{1 \leq i<j \leq Z} \frac{e^{2}}{\left|r_{i}-r_{j}\right|}-\sum_{j=1}^{Z} \frac{Z e^{2}}{\left|r_{j}-r_{0}\right|}
$$

where $r_{0}$ is the nuclear coordinate.

$\tilde{H}$ is specified in the sense that $\hbar, e, m, M$ are names for specific physical parameters with specific real number values. The presence of space variables $r_{j}$ and the state $\Psi$ is taken care of by integration over the space variables and requiring that $\Psi$ is the ground state. In more formal detail the prediction is in terms of a theoretical statement about certain real numbers that must be a theorem of quantum mechanics:

$\exists E \exists \Psi\left\{E=(\Psi, \tilde{H} \Psi) \bigwedge\left[\left(\Psi,(\tilde{H}-E)^{2} \Psi\right)=0\right] \bigwedge \forall E^{\prime}\left(E^{\prime}=(\Psi, \tilde{H} \Psi) \Rightarrow E \leq E^{\prime}\right)\right\}$. 
This statement says that there exists a real number $E$ and a state $\Psi$ where $E=(\Psi, \tilde{H} \Psi)$ and $\Psi$ is dispersion free for $\tilde{H}$ (an eigenstate) and $E$ is the smallest value of $(\Psi, \tilde{H} \Psi)$.

At this point one realizes there is a disconnect between theory and experiment. Theory provides a name of a real number as a solution of an equation with auxiliary conditions to specify which solution, if necessary. However experiment gives a finite string of digits in some representation (binary, etc.) that represents a number. This follows from the observation that each experiment yields an apparatus in a final state, part of which is interpreted as a finite digit string.

This is the case for both digital and analog outcomes since for analog outcomes one must read a pointer position on a dial relative to background marks. The fact that the string is necessarily finite is accounted for by saying that the measurement outcome is an "approximation" of the true value, or that it is a consequence of the finite precision and accuracy of the measurement procedure.

There are two problems here: One is that the measurement outcome is an approximation of a real number and is not a real number. The other follows from the observation that the measurement outcome, as a finite digit string, is considered to be an initial string of an infinite digit string that corresponds to the outcome in the ideal limit of infinite precision and accuracy. However this is quite a different type of name than that provided by theory which names a real number by an equation for which it is a solution.

This disconnect is bridged by the use of computers. Here the purpose of a computer is to convert or translate a real number name as a solution of an equation to a finite digit string that is an approximation to the real number solution. This digit string can be directly compared to the measurement outcome to look for agreement or disagreement.

As is well known much effort in physics goes into computing approximate solutions to theory equations that are difficult to solve. Here consideration will be limited briefly to two aspects. One is that, as a physical dynamical operation with physical systems, a computation is based on arithmetic operations on finite digit strings, This arithmetic, called computer arithmetic, is much studiedł ${ }^{46}$. Differences between computer arithmetic and real number arithmetic include the use of roundoff computations to minimize the effects of working with finite digit strings. The computation output is also a finite digit string that is supposed to be an approximation to an infinite digit string real number.

The other aspect relates to the correspondence between the equation to be solved and a particular computer. If the standard Turing machine representation is used the correspondence associates one or more Turing machine names with the equation to be solved. The same holds for any other standard representation.

The main point to make here is that the determination of this map or correspondence is often nontrivial. In any case, the details of the map do not seem to be part of the physical theory. Instead the map seems to be part of the metatheory and language used to describe the physical theory and its mathematics. A goal, which is open at present, is to extend the theory so that it can at least describe and may possibly prove which computations (Turing machine names) 
will solve a specified equation to a specified approximation.

\subsection{Real Numbers and Outcomes of Measurements and Computations}

The goal here is to examine the relation between outcomes of measurements and computations, and real numbers. It will be seen that there are problems that do not seem to be adequately treated so far.

To begin it is to be emphasized that the numbers of interest in a theory such a quantum mechanics are real and complex numbers. This is based on the mathematical structure of quantum mechanics as state spaces, operator algebras. and other mathematical systems all based on the field $C$ of complex numbers. The elements of $C$ are often shown as pairs $(r, i)$ of real numbers denoting the real and imaginary components. Real numbers can also be described as a subfield $R$ of $C$. Rational numbers are present as a subfield $R a$ of $R$, integers are a subring of $R a$, and the natural numbers are the nonnegative integers.

As is well known, rational numbers, integers, and natural numbers are present as special types of real numbers. In a $k$ - ary representation each rational number is represented as an infinite string of $k$-ary digits whose tail is an infinite repetition of some finite digit string. Rational string numbers are a restriction of rational real numbers to strings whose tail is an infinite string of zeros. Integers, and natural numbers have a similar representation where the tail of zeros begins at the " $k-a l$ " point. Binary examples of the four types are respectively, $-11001.01(011)(011)(011) \cdots, 1011.110110000 \cdots$, $-11101.000 \cdots, 1101.000 \cdots$.

However outcomes of measurements and of computations are, physically, states of parts of a system. For computers or measurement equipment they are states of some output display such as a liquid crystal display or an output register. Quantum mechanically the states of these output parts can be represented by density operators obtained by tracing over other system degrees of freedom including the environment if needed.

As is well known these output states of computations or measurements are interpreted as numbers represented by finite strings of digits or, using the language of information theory, as product states of finite strings of kits or qukits ( $k$ - ary representation). The fact that these strings are finite is based in part on the physical limitation that infinite output strings are impossible to create or read in a finite amount of space and time. Also equally well known are the finite accuracy and precision associated with all computations and measurements. This is often expressed by referring to measurement outcomes as good to $n$ significant figures.

The problem of how one relates computation or measurement outcomes as finite strings to real numbers is well known. This is especially so for measurement of observables with continuous spectra such as position and momentum.

One approach to the problem is the use of coarse graining. In this case assume that the computer or measurement equipment has $n$ binary significant 
figure outputs over some range from $a$ to $b$. Coarse graining assumes that each such output represents a bin or range of real numbers. For example for $n=5$ the output 11.011 might represent all real numbers $r$ where $11.0101000 \cdots \leq$ $r \leq 11.0111000 \cdots$.

Of course many different binnings are possible. More generally if the output is represented by $\underline{s} . \underline{t}$ (fixed point representation) the number of significant figures is given by $L(\underline{s})+L(\underline{t})$ where $\underline{s}$ and $\underline{t}$ are binary strings of respective lengths $L(\underline{s})$ and $L(\underline{t})$. Then one can represent a coarse graining by the choice of two real numbers $\Delta_{l}$ and $\Delta_{u}$ where the output $\underline{s} . \underline{t}$ represents all real numbers $r$ where $\underline{s} . \underline{t} 000 \cdots-\Delta_{l} \leq r \leq \underline{s} . \underline{t} 000 \cdots+\Delta_{u}$. Many choices are possible for the values of $\Delta_{l}$ and $\Delta_{l}$. They can be set independent of $\underline{s} . \underline{t}$, or they may depend on $\underline{s} . \underline{\text {. }}$. Other types of maps are statistical with a chosen probability distribution over different binnings.

This exposes the problem of how one relates an $n$ significant figure output of a computation or measurement to the real numbers. Some type of map is clearly needed for comparison of theory with experiment for any theory as a mathematical structure over $C$, such as quantum mechanics. The map should apply for arbitrary values of $n$ as different experimental and computational setups can give different values of $n$.

This is not a new problem. Recent work to treat this problemt ${ }^{48}$ separates the Hilbert space of quantum mechanics into a product space $\mathcal{H}_{\text {coarse }} \otimes \mathcal{H}_{\text {fine }}$ where states in $\mathcal{H}_{\text {fine }}$ are not observable. Other work $149^{\circ}$ is based on the use of state vectors with rational components. This has been criticized as being inconsistent with axioms of geometryt ${ }^{50}$.

It should be emphasized that for essentially all experiments done to date, which coarse graining is chosen does not affect the comparison of theory and experiment. If it did this would have been discovered by now. However the method of connecting $n$ significant figure outputs of measurements and computations to theory is a question of some concern, particularly if one is interested in foundational aspects of the relationship between physics and mathematics.

\subsection{Connecting $n$ Figure Outcomes of Measurement and Computation to Theory}

An important aspect of a coherent theory of physics and mathematics is that it be very closely connected to computations and experiment. One method to consider is to tie the theory in to what is actually done in experiments and computation. The method proposed here is to replace a physical theory $T$, as a mathematical structure based on $C$ by a theory $T h_{n}$ based on $C_{n}$.

The idea is to let $C_{n}$ be a set of numbers with associated arithmetic relations that more closely ties in with the properties of computation and measurement outcomes than $C$ does. In this case experimental and computational support for $T h_{n}$ would be based on computations and measurements with outcomes in $C_{n}$. In essence these are numbers given to at most $n$ significant figures in a $k$-ary representation for a fixed $k$ value. As such they more closely represent 
measurement and computational outcomes than do the numbers in $C$.

Measurement and computations with $m$ significant figure outcomes are included in $T h_{n}$ if $m \leq n$. In this case a coarse graining would be needed to map the $m$ significant figure outcomes into $C_{n}$. However such a coarse graining, which is from $m$ significant figure numbers to $n$ significant figure numbers, is easier to accept than one into $C$. Also the numbers in $C_{n}$ and the outcome numbers are of the same type in that they are both finite length string numbers..

Measurement and computation outcomes with $m>n$ are not in the domain of $T h_{n}$ as the outcomes are not in $C_{n}$. These belong to all theories $T h_{\ell}$ where $\ell \geq m$. This shows that one must consider the sequence of theories $T h_{n}$ with increasing $n$ along with the $\operatorname{limit}_{n \rightarrow \infty} T \lim _{n}$. How the limit is taken is a matter of great importance. It would be expected that the limiting process is such that the limit theory includes much, or almost all, of the existing $C$ based physical theory. This is necessary because of the great success that $C$ based physical theories have had to date. Also this approach may shed new light on foundational aspects and help solve basic open problems in physicst ${ }^{6}$.

There are several candidate definitions for the numbers in $C_{n}$. One is based on the floating point arithmetic used in computations. Each complex number in $C_{n}$ is a pair of string number pairs, $\left\{\left( \pm \underline{s}_{r}, \pm \underline{e}_{r}\right),\left( \pm \underline{s}_{i}, \pm \underline{e}_{i}\right)\right\}$. The number 0 is included as $\left(\underline{0}_{[1, n]}, \underline{0}\right)$. The subscripts $r, i$ denote the real and imaginary components. For a $k$-ary representation $\underline{s}$ denotes a string of $n k$ basis digits where the leftmost digit $\underline{s}_{1} \neq 0$ and the $" k-a l$ point follows the righthand most digit, $\underline{s}_{n}$. The subscripted brackets $[a, b]$ denote place labels ranging from $a$ to $b$. Here $\pm \underline{e}$ denotes a $k$ basis representation of integral powers of $k$.

As an $n$ significant figure representation of numbers, these string pairs correspond to the number representations used by computers both during computation and as input and output. For example all computers are based on $n$ bit precision arithmetic and treat numbers within some very large range. This is shown by the IEEE floating point arithmetic standards used by most computers. For example some computers use 32 bit single precision arithmetic on all numbers with magnitude $m$ between $10^{+30}$ and $10^{-30}$, or 64 bit double precision arithmetic on all numbers between $10^{+300}$ and $10^{-300}$.

A slightly different, but equivalent form of number is used to report experimental values of physical quantities. These are often given as $\underline{s}_{1} \cdot \underline{s}_{[2, n]} \times 10 \underline{e}$ where $\underline{s}_{1} \neq 0$. Often an uncertainty $\pm 0 . \underline{0}_{[2, n-1]} a_{n} a_{n+1} \times 10 \underline{e}$ is associated with the value.

The importance of $n$ significant figure numbers to experiments is also shown by the observation that in general the magnitude of the error of a measurement is proportional to the magnitude of the quantity being measured. Even more important is the fact that the proportionality factor is almost independent of the type and magnitude of the measured parameter. In the language used here this means that for most experimental values of physical quantities, the value of $n$ is almost independent of the magnitude of the quantity being measured. For example, for most distance measurements $2 \leq n \leq \sim 10$ for distance measurements over a range of $10^{31}$, from nanometers to millions of light years.

The type of representation chosen here is based on outcomes of continuous 
value parameter measurements such as those for space or momentum. These are recorded on a set of $n$ dials or registers where each dial or register records the digits $0-9$ (decimal). The readings $\underline{0}_{[1, n]}, \underline{0}_{[1, n-1]} 1_{n}$, and $\underline{9}_{[1, n]}$ correspond to nondetect, threshold detection (at the sensitivity level of the equipment), and the maximum value possible for the equipment used. This type of equipment is in wide use in flow measuring meters as in utility electric and gas meters.

This describes measurement outcomes for just one apparatus over a finite range from nondetect to detect to maximum. The whole range of quantity values from 0 to $\infty$ can be covered by an infinite hierarchy of measurement apparatuses where just off scale for the $j t h$ apparatus corresponds to detection for the $j+1 s t$. The hierarchy, which is infinite in both directions, can be represented schematically in binary as

$$
\begin{aligned}
& \begin{array}{lllll}
\infty \cdots & j+1 & j & j-1 & \cdots-\infty
\end{array} \\
& \left\{\begin{array}{c}
\text { nondetect } \\
\underline{0}_{[1, n]} .
\end{array}\right\} \quad \underline{1}_{[1, n]} . \quad \quad \text { off scale } \\
& \underline{0}_{[1, n-1]} 1_{n} . \quad\left\{\begin{array}{c}
\text { off scale } \\
\underline{1}_{[1, n]} \cdot+\underline{0}_{[1, n-1]} 1_{n} .
\end{array}\right\} \quad \text { offscale } \\
& \text { nondetect nondetect } \underline{1}_{[1, n]} \text {. }
\end{aligned}
$$

The hierarchy suggests a representation in the form $\left( \pm_{\underline{s}}[1, n] ., \pm \underline{n e}\right)$ where $\underline{s}_{[1, n]} . \neq \underline{0}_{[1, n]}$. if $\underline{e} \neq \underline{0}$ and $\underline{e}$ is any integer. Note that the exponent $\underline{n e}=n \underline{e}$ depends on $n$ and $\underline{e}$. Here the use of significant figure is different from usual in that all $0 s$ to the left of nonzeros, as in 0010, are significant. This is based on the significance of the leftmost $0 s$ in this type of measurement. The "bi-nal" point to the right is also to be noted.

For reasons that will become clear later on, a symmetric form of these numbers is much better. This form corresponds to the strings

$$
\left( \pm \underline{s}_{[1, m]}^{\prime} \cdot, m\left(\underline{e}-\frac{1}{2}\right)\right)=\left(\underline{s}_{[1, n]} \cdot \underline{t}_{[1, n]}, 2 n \underline{e}\right)
$$

Here $m=2 n$ and $\underline{s}_{[1, m]}^{\prime}=\underline{s}_{[1, n]} * \underline{t}_{[1, n]}$ where $*$ denotes concatenation. This representation is valid for any basis $k$. Here emphasis is placed on a binary basis $k=2$ although sometimes a decimal basis is used for illustration.

The ordering of these numbers can be expressed as an infinite alternating sequence of $2^{2 n}-1$ numbers with constant spacing equal to $1 \times 2^{-2 n(\underline{e}-1 / 2)}$. These regions are separated by exponential jumps of $2^{2 n}$, where $\underline{e} \rightarrow \underline{e \pm 1}$. Note that there is no least or greatest number. Here is an example for $n=2$.

$$
\begin{aligned}
& 00.01,00.10, \cdots, 10.00,10.01, \cdots, 11.11 \times 2^{4(\underline{e}+1)} \\
& 00.01,00.10, \cdots, 10.00,10.01, \cdots, 11.11 \times 2^{4 \underline{e}} \\
& 00.01,00.10, \cdots, 10.00,10.01, \cdots, 11.11 \times 2^{4(\underline{e}-1)}
\end{aligned}
$$

Arithmetic with these numbers is is interesting as it combines both region and jump arithmetic. Region arithmetic, which applies in the regions with 
$2^{2 n}-1$ steps of constant spacing, is the same as computer arithmetic except that the range over which the arithmetic applies is proportional to string length instead of being independent of it. An example would be $n$ bit arithmetic over a range of $10^{ \pm n c}$ where $c$ is some constant.

Jump arithmetic consists of arithmetic operations between numbers in different regions or which take numbers in one region to those in another. It also depends on the significance of leading or trailing 0 strings. Here are some examples for $m=2 n=4$.

$$
\begin{aligned}
& 00.10 \times 2^{8}+{ }_{4} 00.10 \times 2^{4}={ }_{4} 00.10 \times 2^{8} \\
& 10.10 \times 2^{4}+{ }_{4} 11.10 \times 2^{4}={ }_{4} 00.01 \times 2^{8} \\
& 11.10 \times 2^{8} \times{ }_{4} 10.01 \times 2^{4}={ }_{4} 00.01 \times 2^{16}
\end{aligned}
$$

Extensive roundoff has been used in these examples. $2 n=4$ has been used for the subscripts $m$ on $={ }_{m},+_{m}, \times_{m}$. String $a b . c d={ }_{4} a^{\prime} b^{\prime} \cdot c^{\prime} d^{\prime}$ if the corresponding primed and unprimed digits are the same.

The reader may wonder why one has to contend with both region and jump arithmetic when computer arithmetic used in physics is limited to the region type. The reason is that for low values of $n$ extensive roundoff is needed and arithmetic operations often take numbers from one region to another. ${ }^{4}$ As $n$ increases region arithmetic increasingly dominates. For large $n$ the need for jump arithmetic decreases until it disappears in the limit $n \rightarrow \infty$. The reason for this will be seen later on.

Here the set of complex string numbers $C_{n}=\left\{R_{n}, I_{n}\right\}$ where $R_{n}$ and $I_{n}$ each consist of the set of all string numbers in the form shown by Eq. 2 There is no bound on the magnitude of the numbers in $R_{n}$ (and $I_{n}$ ) as the length, $L(\underline{e})$, of the exponent is unbounded.

Before applying these ideas to any theory based on these numbers it is worth emphasizing several aspects. One is that mathematical aspects of the theory are based on the numbers in $C_{n}$. No other numbers are available for the theory. For instance, arithmetic equality for two numbers $\left( \pm \underline{s}_{[1, n]} \cdot \underline{t}_{[1, n]}, \pm 2 n \underline{e}\right)$ and $\left( \pm \underline{s}_{[1, n]}^{\prime} \cdot \underline{t}_{[1, n]}^{\prime}, \pm 2 n \underline{e^{\prime}}\right)$ expressed by

$$
\left( \pm \underline{s}_{[1, n]} \cdot \underline{t}_{[1, n]}, \pm 2 n \underline{e}\right)=_{2 n}\left( \pm \underline{s}_{[1, n]}^{\prime} \cdot \underline{t}_{[1, n]}^{\prime}, \pm 2 n \underline{e^{\prime}}\right),
$$

holds if the signs are the same and $\underline{s}=\underline{s}^{\prime}, \underline{t}=\underline{t}^{\prime}$, and $\underline{e}=\underline{e}^{\prime}$. Note the different uses of equality. The arithmetic definition, $=_{2 n}$, depends on $n$. Two strings are equal if they are identical as strings.

All the other arithmetic operations are also subscripted by $2 n$ as they depend on $n$. Examples are (subscripts $[1, n]$ are suppressed)

$$
(-\underline{s} \cdot \underline{.}, 2 n \underline{e})+{ }_{2 n}\left(+\underline{s}^{\prime} \cdot \underline{t}^{\prime}, 2 n \underline{e^{\prime}}\right){ }_{2 n}\left(+\underline{s}^{\prime \prime} t^{\prime \prime}, 2 n \underline{e^{\prime \prime}}\right)
$$

and

$$
(-\underline{s} \cdot \underline{t}, 2 n \underline{e}) \times_{2 n}\left(+\underline{s}^{\prime} \cdot \underline{t}^{\prime}, 2 n \underline{e^{\prime}}\right)=2 n\left(+\underline{s}^{\prime \prime} t^{\prime \prime}, 2 n \underline{e^{\prime \prime}}\right) .
$$

\footnotetext{
${ }^{4}$ Consider for example binary arithmetic as done on a computer based on 4 bit strings.
} 
Here $+_{2 n}$ and $\times_{2 n}$ include roundoff operations in their definitions.

The other aspect of $T h_{n}$ is that, at first sight, one can think of many reasons why theories based on $C_{n}$ instead of the usual complex number continuum $C=C_{\infty}$ are not useful. Examples include the loss of closure or completeness properties for components of the mathematical structure over $C_{n}$, the explicit $k$ dependence of the definitions of $=_{n}$ and the arithmetic operations, and the specific method of roundoff. Also because of roundoff, addition is not associative.

As is well known from the operations of computers based on computer arithmetic, these aspects do not cause problems for almost all operations. For axiomatizable properties such as associativity, the usual axiom of unconditional associativity would be replaced with a conditional statement that if numbers in a triple have certain specific properties, then addition $\left(+_{n}\right)$ is associative.

What is important, though, is that these undesirable properties disappear in some fashion as $n \rightarrow \infty$. How this happens depends on the property and how the limit is taken. For example, the importance of roundoff decreases as the length of bit strings used in a computation increases relative to the length of the input and output strings. Possible meanings of disappearance include the idea that the probability of states appearing in the theory experiment connection with one or more of these undesirable properties goes to 0 as $n \rightarrow \infty$. Details on this must await additional work especially on how the approach of $n$ to $\infty$ is taken.

\section{$4 \quad R_{n}$ Based Space and Time}

The structure of $R_{n}$ space and time is different from that based on $R$. The reason is that the only numbers available are those in $R_{n}$. The numbers in $R_{n}$ are taken here to have the form $\left( \pm \underline{s}_{[1, n]} \cdot \underline{t}_{[1, n]}, \pm \underline{2 n e}\right)$ where $\underline{s}_{[1, n]} \cdot \underline{t}_{[1, n]} \neq \underline{0}_{[1, n]} \cdot \underline{0}_{[1, n]}$. This form (Subsection 3.4) will be used as it has the right limit properties. The number 0 is added as the string $\left(\underline{0}_{[1, n]} \cdot \underline{0}_{[1, n]}, 0\right)$.

Two aspects need emphasis. The properties of space and time, as shown in the figures, look very different from the usual continuum space and time. This is a consequence of the facts that the differences are greatest for small values of $n$, and it is possible to create illustrative figures for small values of $n$ only. Also one must keep in mind that the figures are drawn on a usual continuum based background. The background space is not part of space or time in a theory such as $Q m_{n}$. It is instead included in $C$ based physics, such as $Q m=Q m_{\infty}$. This emphasizes the importance of taking the limit $n \rightarrow \infty$. The fact that $C_{n}$ based space and time looks strange for finite $n$ is not important. What is important is that a continuum space and time that is locally flat be recovered in the limit $n \rightarrow \infty$.

In what follows, the \pm sign on $\underline{2 n e}$ is assumed to be included in $\underline{e}$. Thus $\underline{e}$ is a $k$-ary string representing an integer and $\underline{2 n e}=2 n \times \underline{e}$ represents ordinary integer multiplication. The forms $\underline{2 n e}$ or $2 n \underline{e}$ are interchangeable as they are equivalent.

The properties of $R_{n}$ based space can be seen by showing the point locations in one space dimension. This is shown in Figure 1 for $n=1$ and $k=2$ (binary) 


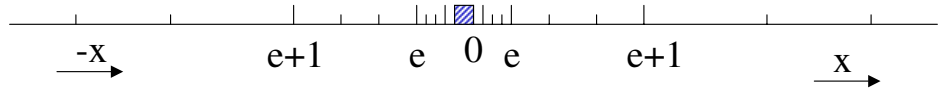

Figure 1: Coordinates for one Space Dimension for Positive and Negative $x$ Values for $k=2 n=2$. The jump locations are shown by $e$ and $e+1$. The crowding towards $x=0$ is shown by parallel line shading.

for positive and negative values of $x$. The allowed point locations are shown by the vertical ticks along the line.

Probably the most interesting aspect of the figure is the crowding together of the points as one approaches the $x=0$ point. The rate of crowding or decrease in neighborhood spacing can be best described as equal spacing punctuated by exponential decreases or increases as one moves toward or away from the origin at 0 which is an accumulation point. The shading at 0 represents the crowding of the points. As is shown in the figure for $2 n=2=k$, for each $e$ there are $2^{2 n}-1$ intervals of spacing $2^{2 n(e-1 / 2)}$. Then the interval spacing changes to $2^{2 n(e-1 / 2 \pm 1)}$ for the next $2^{2 n}-1$ intervals. As $e$ is not bounded there is no lower or upper bound on the interval size.

This constancy of neighboring point distances punctuated by exponential jumps holds in $R_{n}$ for any value of $n$ and $k$. For each value of $\underline{e}$, there are $k^{2 n}-1$ successive locations each separated by a distance of $k^{2 n}(\underline{e}-1 / 2)$. The separation distance jumps to $k^{2 n(\underline{e}+1 / 2)}$ as $\underline{s}_{[1, n]} \cdot \underline{t}_{[1, n]}$ goes from $\underline{1}_{[1, n]} \cdot \underline{t}_{[1, n]}$ to $\underline{0}_{[1, n]} \cdot \underline{.}_{[1, n-1]} 1_{n}$ In the opposite direction the separation distance drops to

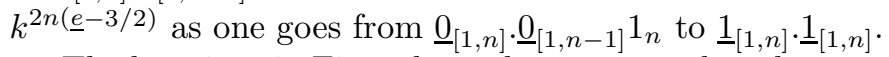

The locations in Fig. 1 1 have the property that they are scale invariant under multiplication by an integral power of $2^{2 n}$. The multiplication is in effect an exponent translation by replacing $e$ by $e+j$ where $j$ is any integer. This is quite different than the usual lattice used in physics (e.g. lattice gauge theory). There the system is invariant under addition of an integral number of unit intervals.

Figure 2 extends Fig 11 to two space dimensions in Cartesian coordinates. The space point locations, which are discrete, correspond to the intersections points of lines parallel to the $x$ and $y$ axes. The crowding of the lines towards the axes shows that points on the axes are accumulation points. These are denoted by circles on the axes.

The figure shows that for each value of $y, x=0$ is an accumulation point from either $x$ direction. Similarly for each value of $x, y=0$ is an accumulation point for $y$ values from either direction. The origin is an accumulation point for 


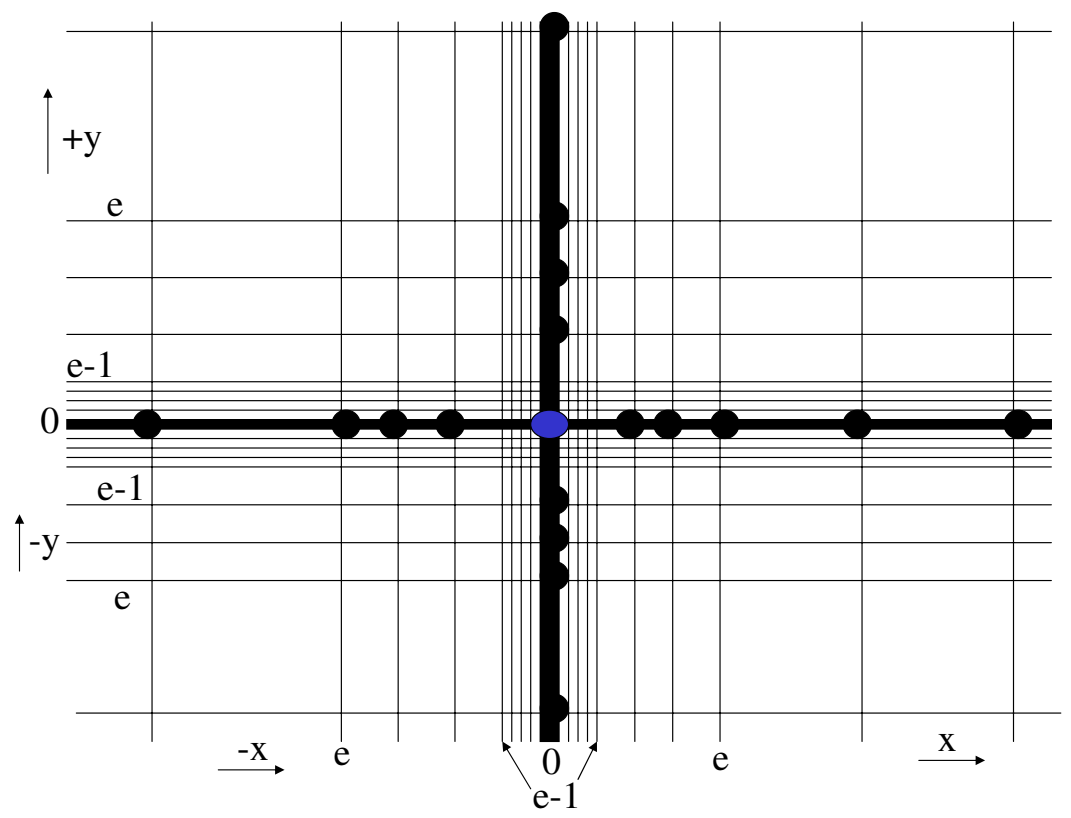

Figure 2: Coordinates for two Space Dimensions for all $R_{n}$ Values of $x$ and $y$. The intersections of the grid lines show the allowed space locations. The jump locations are given by $e$ and $e-1$. The crowding towards the $x=0$ and $y=0$ axes is shown by parallel line shading. Circles on the axes and at the origin denote the points of accumulation.

both $x$ and $y$ values.

Extension of this description to three dimensions is straightforward. There one has three types of accumulation points, the origin which is a three dimensional point of accumulation, points on the $x, y$, and $z$ axes which are two dimensional accumulation points, and points on the $x-y, x-z$, and $y-x$ planes which are one dimensional.

The proof of this singular nature of the points of accumulation rests on the observation that, unlike other points, they do not have nearest neighbors in one or more dimensions. The origin has no nearest neighbors in any dimension. The points on the axes have nearest neighbors in one direction and not in the other. Points on the coordinate planes in three dimensional space have nearest neighbors in two dimensions but not in the third.

As is well known ordinary flat space and the usual lattice representations are invariant under translations. Scale transformations play the same role for the space described here in that it is invariant under scale "translations". For the representation used here for each value of $k$ and $n$ the scale transformation corresponds to any translation of the exponent $\underline{e}$ in the number $\left(\underline{s}_{[1, n]} \cdot \underline{t}_{[1, n]}, 2 n \underline{e}\right)$. This corresponds to replacing $\underline{e}$ by $\underline{e}+j$ where $j$ is any integer and gives a scale change by a factor of $2^{2 n j}$.

The effect of this on the space coordinates is remarkable. It corresponds 


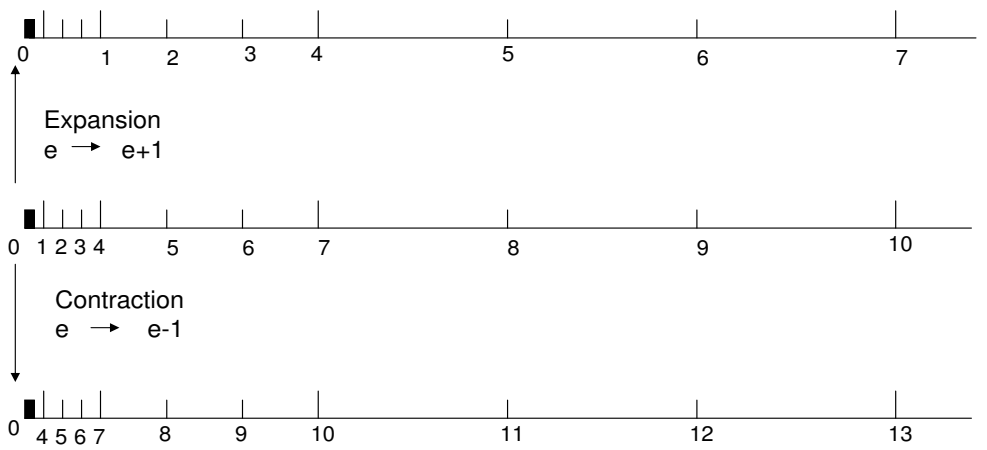

Figure 3: An example of scale transformations showing expansion $j=+1$ (top figure) and compression $j=-1$ (bottom figure) in one dimension for $k=2 n=2$ and positive values. The numbers under the tick marks show the effect of the transformations on individual points. The origin at the short shaded rectangle is left unchanged with the region around the origin serving as an infinite source of points for expansion and an infinite sink for compression. The corresponding figure for negative values is obtained by reflection through the origin.

to an expansion or stretch for $j>0$ and a contraction for $j<0$. The origin or space singularity is unchanged and is fixed. This is shown in Figure 3 for one space dimension for positive valued locations for $j=+1$ and $j=-1$ with $k=2 n=2$. The expansion and contraction for negative valued locations is obtained by reflecting the figure through the origin.

The fixed location of the origin which is a singularity in space follows because multiplying it by any scale transformation still gives 0 . Also what is remarkable is that the immediate neighborhood around the singularity serves as an infinite source of points for expansions and an infinite sink for points for contractions. This is shown schematically in the figure by the appearance of unlabelled points in the top figure and the disappearance of points in the bottom figure.

These transformations can easily be extended to 2 and 3 space dimensions. In this case if the scale transformations are the same in each dimension then the shape of a figure or object is preserved. Only its size is changed. However if they are different in different dimensions then the shape of a figure is changed.

The allowed values of time have similar properties to the $x$ values shown in Fig. 1] The values crowd toward the accumulation point at 0 . In nonrelativistic quantum mechanics where time is simply a parameter for describing dynamic evolution, one would expect that all numbers in $R_{n}$, including 0 , should be possible values of $t$. However it will be seen later on that a description of dynamics in terms of step operator iteration can never reach 0 . Also time and space are treated on an equal footing as coordinates in relativistic physics. In this case $t=0$ is excluded for the same reasons as were given for exclusion of $x=0$ in that the metric distance between 0 and any allowed value of $t$ is not a number in $R_{n}$.

The locations shown in Figures 10 2 and 3] also illustrate the natural ordering of the points in in each space dimension. The ordering is related to arithmetic 
ordering based on iteration of the ordinary +1 operation in arithmetic punctuated by exponential jumps. The relation between the two is given by a basic ordering step function $f_{<}$defined on $R_{n}$ by

$$
f_{<}(\underline{s} \cdot \underline{t}, 2 n \underline{e})= \begin{cases}(\underline{s} \cdot \underline{t}, 2 n \underline{e})+2 n\left(\underline{0} \cdot \underline{0}_{[1, n-1]} 1_{n}, 2 n \underline{e}\right) & \text { if } \underline{s} \cdot \underline{t} \neq \underline{1} \cdot \underline{1} \\ \left.\left(\underline{0} \cdot \underline{0}_{[1, n-1]} 1_{n}, 2 n \underline{e}+1\right)\right) & \text { if } \underline{s} \cdot \underline{t}=\underline{1} \cdot \underline{1}\end{cases}
$$

This definition is for positive $\underline{s}_{[1,2 n]}$. The subscripts $[1, n]$ have been suppressed for brevity. Note that $\underline{e}$ can be positive or negative and is unbounded. Also $f_{<}$ is not defined on $(\underline{0} \cdot \underline{0}, \underline{0})$, the 0 location.

The inverse operation $f_{>}=f_{<}^{-1}$ can be explicitly defined. Or it can be defined from $f_{<}$by

$$
f_{>}\left(f_{<}(\underline{s} \cdot \underline{t}, 2 n \underline{e})={ }_{2 n}(\underline{s} . \underline{t}, 2 n \underline{e}) .\right.
$$

Note that $(\underline{0} . \underline{0}, 0)$ is not in the range of $f_{>}$.

The definition of $f_{<}$on negative $\underline{s}$ is defined by

$$
f_{<}\left(-\underline{s} \cdot \underline{t}, 2 n(\underline{e})=-\left(f_{>}(\underline{s} \cdot \underline{t}, 2 n \underline{e})\right) .\right.
$$

This says that moving along with the ordering on negative numbers is equivalent to moving opposite to the ordering on the positive numbers and changing the sign.

What is interesting here is that the origin or point $\left(\underline{0}_{[1, n]} \cdot \underline{0}_{[1, n]}, 0\right)$ in any dimension is unreachable by the iterative action of $f_{>}$on $R_{n}$. Applied to motion in a coordinate system, this iterative action corresponds to moving backward in basic elementary steps towards the origin in either the space or time directions. Repeated iterations take one arbitrarily close but never reach the origin.

This property, and the fact that the location is unchanged under expansions and contractions reminds one of the big bang in cosmology. The big bang has the property that one can go backwards in time and get arbitrarily close, but never reach it. Also similarities between the expansion of the universe and the coordinate system expansion shown in Fig. 3 are of interest. ${ }^{5}$

Further development in this direction must await further work. One should note that the proof of the uniqueness of the origin fails for $R$ based space and time. It follows that $R$ based space and time shows no difference between the origin and other points. This is an illustration of the possibility that the description of space and time, obtained by taking the limit $n \rightarrow \infty$, can be different from that based directly on $R$ at the outset.

\section{Quantum Mechanics Based on $C_{n}$}

Perhaps the first point to note about $Q m_{n}$, which is quantum mechanics based on $C_{n}$, is that spaces $\mathcal{H}$ are preHilbert spacest ${ }^{51}$. They are not Hilbert spaces

\footnotetext{
${ }^{5}$ It is tempting to associate galactic black holes with the singularities on the axes for some of the degrees of freedom in spaces with two or more degrees of freedom. Examples in 2 dimensional space are the points with $x=0$ and $y \neq 0$ or conversely. These points move under expansions and contractions. Future work will determine if the rigidity of their locations on straight line axes can be relaxed.
} 
as they are not norm complete. This follows from the facts that numerical coefficients of states are numbers in $C_{n}$ and that the definition of the bilinear functional or scalar product $(\psi, \phi)$ is from pairs of states in $\mathcal{H}$ to $C_{n}$.

It is also the case that all operators over $\mathcal{H}$ in $Q m_{n}$ are discrete in that their spectra are subsets of $C_{n}$. This follows directly from the discreteness of the elements of $C_{n}$. Thus all operators can be expanded as a sum over eigenvalues and eigenstates or eigenspaces as $\tilde{A}=\sum_{a} a \tilde{P}_{a}$ where $a$ is in $C_{n}$ and $\tilde{P}_{a}$ is a projection operator over a subspace of $\mathcal{H}$.

It is important in discussing states and properties of states to keep the various meanings of symbols separate. Thus in writing $\psi=c_{a}|a\rangle+c_{b}|b\rangle,=$ and + have their usual meaning. But the orthonormality of the basis states uses $={ }_{2 n}$ as in $\langle a \mid b\rangle={ }_{2 n} \delta_{a, b}^{n}$. Here $\delta_{a, b}^{2 n}={ }_{2 n} 1[0]$ if $a=_{2 n}\left[\neq_{2 n}\right] b$ where 1 and 0 are their representations, $\left(\underline{0}_{[1, n-1]} 1_{n} \cdot \underline{0}_{[1, n]}, 0\right)$ and $\left(\underline{0}_{[1, n]} \cdot \underline{0}_{[1, n]}, 0\right)$, in $C_{n}$. Note that for digit strings in $C_{n}$ the least and most significant subscript labels are $n$ and 1 respectively. Also as is usual the more significant digits stand to the left of less significant ones.

Note that here one takes seriously the use of natural numbers, integers and even rational numbers as stand ins or short names for the corresponding numbers in either $C$ or $C_{n}$. Thus 1 which appears in the normalization condition is a name in $C$ for $1 . \underline{0}_{[2, \infty]}=\lim _{\ell \rightarrow \infty} 1 \cdot \underline{0}_{[2, \ell]}$. Since real numbers can be considered as equivalence classes of convergent Cauchy sequences of rational string numbers, either $1 . \underline{0}_{[2, \infty]}$ or $\lim _{\ell \rightarrow \infty} 1 . \underline{0}_{[2, \ell]}$ are names for two specific equivalence classes.

The normalization condition for states such as $\psi=c_{a}|a\rangle+c_{b}|b\rangle$ is expressed as

$$
\left|c_{a}\right|_{n}^{2}+{ }_{2 n}\left|c_{a}\right|_{n}^{2}=2 n 1
$$

where $\left|c_{a}\right|_{n}^{2} \equiv c_{a} \times_{2 n} c_{a}^{*}$. It should be noted that in certain cases because of roundoff this relation may not be exact. A good example is the state $a|\alpha\rangle+$ $b|\beta\rangle+c|\gamma\rangle$ with $a={ }_{2 n} b={ }_{2 n} c$ and $|a|_{n}^{2}+{ }_{2 n}|b|_{n}^{2}+{ }_{2 n}|c|_{n}^{2}={ }_{2 n}\left(\underline{0}_{[1, n-1]} 1_{n} \cdot \underline{0}_{[1, n]}, 0\right)$. For $n=2, a={ }_{4}(57.74,-2)=57.74 \times 10^{-2}$ (decimal) with roundup of the fifth figure. With roundup of each square, this gives 1.0002 instead of 1.000. This type of mismatch is well known in computer arithmetic and is why different algorithms with different roundoff procedures are used for different problems. It is also why the value of $n$ in computations is much larger than the number of significant figures in the final outcome.

Here a specific computer arithmetic is assumed for $C_{n}$. As was the case for the $T h_{n}$, for each arithmetic with its specific roundoff prescription and algorithms for implementing $+_{2 n}$ and $\times_{2 n}$ there is a slightly different theory $Q m_{n}$. This is not a problem because as $n$ increases, the number of significant figures needed to see the effects of different roundoffs, etc. becomes inaccessible to experimental or computational outcomes. Thus one would expect that the differences between these theories vanishes in the limit as far as agreement or disagreement between theory and experiment is concerned.

$R_{n}$ based space and time is the space and time arena for $Q m_{n}$. So position operators and transformation operators need to reflect the properties of $R_{n}$ space and time. In each dimension the position operator, $\tilde{x}$ has eigenstates 
$\left|x_{ \pm \underline{s}, \underline{e}}\right\rangle$ corresponding to the eigenvalue $x_{ \pm \underline{s}, \underline{e}}$ which is a shorthand notation in $Q m_{n}$ for $\left( \pm_{\underline{s}}[1,2 n] ., 2 n(\underline{e}-1 / 2)\right)$ as a position value. Here $\underline{e}$ includes the sign but not the fixed parameter $n$.

Expansion of a wave packet state in terms of position states in one dimension is given by

$$
\psi=\sum_{e=-\infty}^{\infty} \sum_{\underline{s}}=\left[\left|x_{+\underline{s}, \underline{e}}\right\rangle\left\langle x_{+\underline{s}, \underline{e}} \mid \psi\right\rangle+\left|x_{-\underline{s}, \underline{e}}\right\rangle\left\langle x_{-\underline{s}, \underline{e}} \mid \psi\right\rangle\right] .
$$

The $\underline{s}$ sum is over all $\underline{s}$ such that $\underline{s} \neq \underline{0}_{[1,2 n]}$. Note that the expansion coefficients are all numbers in $C_{n}$.

It should be noted that the choice made here, to exclude singular points from the sum, is arbitrary. The reason is that it is unclear whether the singular points should be included or excluded in such sums over space locations. It is hoped that future work will clarify which choice is right.

This expansion also holds for more than one space dimension. In this case one replaces $\left|x_{ \pm \underline{s}, \underline{e}}\right\rangle$ by $\left|x_{ \pm \underline{s}_{i}, \underline{e}_{i}}\right\rangle$ and sums over the dimension label $i$.

It is tempting to develop $Q M_{n}$ further at this point and describe motion of systems and transformations based on $R_{n}$ space. However, at this point in the development, it is prudent to defer this to future work.

\section{Toward The limit $n=\infty$}

It is useful to reemphasize at the outset the importance of taking the limit and how it is taken. At present many details of the process are not known. However the requirement that continuum space and time be obtained in the limit does guide the process even at this stage of the investigation.

To this end it is useful to look at Eqs. 3 14 and 5 These equations and Figs. 1 and 2 show that there are regions of $2^{2 n}-1$ steps of constant spacing punctuated by exponential jumps. In one direction the spacing intervals are larger by a factor of $2^{2 n}$. In the opposite direction they are smaller by a factor of $2^{-2 n}$. As $n$ increases the number of steps and size of the regions of constant spacing increases exponentially with $n$. The exponential jumps become scarcer but their size increases exponentially with $n$.

It is clear from this that if $R$ based space and time and $C$ based physics is to be recovered in the limit of $n=\infty$, one must have things arranged so that as $n$ increases the positive numbers available to the theory extend from lower and upper limits that approach 0 and $\infty$ respectively as $n$ increases. Also there must be no exponential jumps between these limits, and the spacing between successive numbers must approach 0 as $n$ increases. The same must hold for the negative numbers except that the upper limit of $\infty$ becomes a lower limit of $-\infty$.

These conditions are satisfied by the form, $\left(\underline{s}_{[1, n]} \cdot \underline{t}_{[1, n]}, \pm 2 n(\underline{e})\right.$, of numbers that are used here with $\underline{e}$ set equal to 0 . These numbers range from $2^{-n}$ to $2^{n}$ in steps of size $2^{-n}$. Jumps described by $\underline{e}=0 \rightarrow 1$ and $\underline{e} \rightarrow-1$ occur at 
the upper and lower limits of the range. For negative numbers the ranges are replaced by $-2^{n}$ and $2^{-n}$.

One sees immediately that these numbers satisfy the desired conditions. As $n$ increases the ranges approach 0 and $\pm \infty$ with the spacing going to 0 . Jumps are pushed to 0 from both sides and out to $\pm \infty$ in the limit.

It is easy to see from this discussion why the simpler asymmetric form of the numbers, $\left(\underline{s}_{[1, n]} ., n \underline{e}\right)$ are not satisfactory. The problem is that for $\underline{e}=0$ there is an exponential jump right at the number $1=\left(\underline{0}_{[1, n-1]} 1_{n} ., 0\right)$. Also the location of this jump at 1 is independent of $n$.

\section{Summary and Outlook}

Dissatisfaction with the present state of affairs regarding the foundational relationship between physics and mathematics forms part of the background for this paper. In spite of their obvious close relationship they appear to completely unrelated.

One way to remedy this is to work towards a coherent theory of physics and mathematics. Here work in this direction is based on the physical nature of language and on the connection between theory and experiment. The physical nature of language and numbers leads to the representation of language expressions and numbers as symbol strings that are represented by one dimensional physical systems in different states. For numbers this leads to their representation as strings of digits in some basis (a $k$-ary representation).

Regarding the theory-experiment connection it was noted that the reality status for very small and very large far away objects is more indirect than for nearby laboratory sized objects. This is based on a hierarchal aspect in that an experiment test of a theory already implies the validity of theories needed to ensure that equipment functions properly. The disconnect between theoretical predictions as real number solutions to equations and measurement outcomes as finite number strings of $n$ significant figures is noted. The use of computers to interpolate between the two different types of numbers is noted as is the use of coarse graining to connect experimental numbers to real numbers.

Here a method that ties experiment more closely to theory by reducing the "distance" embodied in coarse graining (connecting numbers with a few significant figures to numbers with an infinite number of figures) is considered. The method consists of replacing the complex numbers on which theories are based by complex numbers as pairs of digit strings of length $n$.

The type of number chosen is based on the outputs of measurement equipment for measuring continuous quantities where the equipment has a threshold sensitivity and a finite range of possible outcomes. These numbers, in the form $\left( \pm \underline{s}_{[1,2 n]}, 2 n(\underline{e}-1 / 2)\right)$ where $\underline{s}_{[1,2 n]}$ is a $k$-ary digit string of length $2 n, \underline{e}$ is a $k$-ary representation of integers and $2 n(\underline{e}-1 / 2)$ is an exponent of $k$, correspond to $2 n$ figure numbers of all possible magnitudes and signs.

Some properties of space and time and quantum mechanics based on these numbers are described. It is seen that space and time points are characterized by 
regions of $k^{2 n}-1$ points with spacing $k^{2 n(e-1 / 2)}$ interspersed with exponential jumps to regions of $k^{2 n}-1$ points with spacing $k^{n(e( \pm 1)-1 / 2)}$.

An interesting aspect of $R_{n}$ space is the classification of points into singular and nonsingular points. In 3 dimensional space the singular points were classified into points that had no nearest neighbors in 1 dimension (coordinate planes), 2 dimensions (coordinate axes), and 3 dimensions, (the origin).

Scale invariant transformations were noted to correspond to expansions and contractions of $R_{n}$ based space by scale factors that are powers of $k^{2 n}$. It was remarkable that all points moved under these transformations except the origin. Also the origin acts like an infinite source and sink for space points for expansions and contractions in all directions.

A few aspects of $Q m_{n}$, quantum mechanics based on $C_{n}$, were described. The fact that all amplitudes and scalar products are required to be numbers in $C_{n}$ was noted. Also the need to use computer type arithmetic with roundoff to describe normalization of states was outlined.

The approach to the limit was discussed briefly. It was seen that in order to approach the continuum limit of $R_{n} \rightarrow R$, the symmetric form $\left(\underline{s}_{[1, n]} \cdot \underline{t}_{[1, n]}, 2 n \underline{e}\right)$ of the numbers was satisfactory in that as $n \rightarrow \infty$ the region of jump free numbers approached 0 and $\pm \infty$ for $\underline{e}=0$.

There is much more work needed to expand on the general proposal outlined here of describing theories such as $Q m_{n}$ and going to the limit $n \rightarrow \infty$. Energy and momentum and many other aspects of physics need to be described. Also more work is needed to support the idea that use of the method outlined here enables space and time to have different properties such as singularities that are invisible to continuum based physics, at least as ab initio properties of space time. Also number representations different from the symmetric one and the one with the " $k-a l "$ point at the end need to be investigated. Finally the important limiting process where one requires that the theory maximally agree with experiment for each $n$ must be investigated.

In conclusion one cannot avoid the speculation that the scale transformations of space corresponding to expansions and contractions are dynamically driven by forces such as gravity and dark energy. This and the possibility of making the parameters $n$ and $\underline{e}$ time dependent will be investigated in future work.

\section{Acknowledgements}

The author thanks Steve Peiper for discussions on Computer Arithmetic. This work was supported by the U.S. Department of Energy, Office of Nuclear Physics, under Contract No. W-31-109-ENG-38.

\section{References}

1. W. H. Zurek, Los Alamos archives preprint quant-ph/0105127 Phys. Rev. D 24 1516, (1981); 261862 (1982). 
2. E. Joos and H. D. Zeh, Zeit. Phys. B 59, 23, (1985); H. D. Zeh quant-ph/9905004 E Joos, quant-ph/9808008

3. S. L. Adler, Los Alamos Archives preprint quant-ph/0112095

4. H. Everett, Reviews of Modern Physics, 29, 454-462(1957);

5. J. A. Wheeler, Reviews of Modern Physics, 29, 463-465, (1957).

6. STRINGS 2000, Proceedings of the 2000 International Superstrings Conference University of Michigan, Ann Arbor 10 - 15 July 2000, edited by Michael J Duff, James T Liu \& Jianxin Lu (University of Michigan), Strings 2000 Conference-Physics Problems for the Next Millenium, URL: http://feynman.physics.lsa.umich.edu/strings2000/millenium.html

7. M. Tegmark, Classical and Quantum Gravity, 14,L69-L75,(19917), Los Alamos Preprint quant-ph/9702052

8. H. van Dam and Y. Jack Ng, Los Alamos Arechives preprint quant-ph/ 0108067 .

9. C. Hogan, Revs. Modern Phys. 72, 1149, (2000).

10. J. D. Barrow and F. J. Tipler, The Anthropic Cosmologic Principle, (Oxford University Press, 1989).

11. S. Weinberg, Dreams of a Final Theory, Vintage Books, New York, NY, 1994.

12. B. Greene, The Elegant Universe, (Vintage Books, New York, NY 2000).

13. M. Tegmark, Ann. Phys. 270, 1-51, (1998).

14. A. A. Frankel, Y. Bar-hillel, A. Levy, and D. van Dalen, Foundations of Set Theory, second revised edition, Studies in Logic and the Foundations of Mathematics, Vol 67, (North-Holland Publishing Co. Amsterdam, 1973).

15. S. Shapiro, Philosophy of Mathematics, Structure and Ontology, Oxford University Press, New York, NY, (1997).

16. A. Heyting, Intuitionism, An Introduction, 3rd Revised Edition (NorthHolland Publishing, New York, 1971).

17. E. Bishop, Foundations of Constructive Analysis (McGraw Hill Book Co. New York, 1967).

18. M. J. Beeson Foundations of Constructive Mathematics Metamathematical Studies, (Springer Verlag, New York, 1985).

19. K. Svozil, Foundations of Physics 251541 (1995); D. Bridges and K. Zvozil, Internat. Jour. Theoret. Phys. 39 503-515,(2000). 
20. P. Benioff, Quantum Information Processing, 1, 495-509, (2002), Preprint quant-ph/0210211

21. R. Landauer, Physics Today 44, No 5, 23, (1991); Physics Letters A 217 188, (1996); in "Feynman and Computation, Exploring the Limits of Computers", A.J.G.Hey, Ed., (Perseus Books, Reading MA, 1998).

22. E. Wigner, Commum. Pure and Applied Math. 13001 (1960), Reprinted in E. Wigner, Symmetries and Reflections, (Indiana Univ. Press, Bloomington IN 1966), pp 222-237.

23. D. Finkelstein, Int. Jour. Theoret. Phys. 311627 (1992); "Quantum sets, assemblies and plexi" in Current Issues in Quantum Logic, E. Beltrametti and B. van Frassen, Eds, (Plenum Publishing Co. New York, 1981), pp 323-333; Quantum Relativity (Springer Verlag, New York, 1994).

24. G. Takeuti, "Quantum Set theory" in Current Issues in Quantum Logic, E. G. Beltrametti and B. C. van Fraasen, Editors (Plenum Press, New York 1981), pp 303-322.

25. S. Titani and H. Kozawa, Int. Jour. Theoret. Phys., 42, pp. 2575-2602, (2003).

26. K-G. Schlesinger, Jour. Math. Phys., 40, pp. 1344-1358, (1999).

27. A. Odlyzko, "Primes, Quantum Chaos and Computers" in Number Theory, Proceeding of a Symposium, May 4 1989, Washington D. C., Board on Mathematical Sciences, National Research Council, 1990, pp 35-46.

28. R. E. Crandall, J. Phys. A: Math. Gen. 296795 (1996).

29. S. Okubo, "Lorentz-invariant Hamiltonian and Reimann Hypothesis" Los Alamos Archives, quant-ph/9707036

30. Y. Ozhigov, "Fast Quantum Verification for the Formulas of Predicate Calculus", Los Alamos Archives Rept No. quant- ph/9809015.

31. H. Buhrman, R. Cleve, and A. Wigderson, "Quantum vs Classical Communication and Computation", Los Alamos Archives Rept. No. quant-ph/9802040

32. D. Spector, Jour. Math. Phys. 39, 1919 (1998).

33. J. Król, Int. Jour. Theoret. Phys., 42, pp. 921-935, (2003); Foundations of Physics, (2004).

34. P. C. W. Davies, "Why is the Physical World so Comprehensible?" in Complexity, Entropy, and Physical Information, Proceedings of the 1988 workshop on complexity, entropy, and the physics of information, may-june 1989, Santa Fe New Mexico, W. H. Zurek, Editor, (Addison-Weseley Publishing Co. Redwood City CA 1990, pp 61-70). 
35. P. Benioff, Phys. Rev A, 58 893-904, (1998).

36. P. Benioff, Foundations of Physics, 32 989-1029, (2002).

37. P. Benioff, Archives preprint quant-ph/0303086

38. A. Peres, Quantum Theory: Concepts and Methods (Kluwer Academic Publishers, Dordrecht, 1993) xiv+446 pp., reprinted in paperback (1995); A. Peres and W. H. Zurek, Is quantum theory universally valid? Am. J. Phys. 50 (1982) 807; A. Peres, The physicists role in physical laws, Found. Phys. 10 (1980) 631; C. A. Fuchs and A. Peres, Quantum theory needs no interpretation, Physics Today 53 (March 2000) 70.

39. J.R. Shoenfield, Mathematical Logic, (Addison Weseley Publishing Co. Inc., Reading, MA, 1967).

40. R. Smullyan, Gödel's Incompleteness Theorems (Oxford University Press, New York, 1992).

41. C. C. Chang and H. J. Keisler, Model Theory, Studies in Logic and the Foundations of Mathematics, Vol 73, (American Elsevier Publishing Co. Inc. New York, NY (1973)).

42. H. Rogers, Theory of Recursive Functions and Effective Computability, McGraw Hill, New York, 1967.

43. J. Väänänen, Bull. Symbolic Logic, 7, pp 504-519, (2001).

44. D. Deutsch, The Fabric of Reality, Penguin Books, New York, 1997.

45. V. W. Marek and J. Mycielski, Amer. Math. Monthly, 108, pp. 449-468, (2001).

46. The extent of study can be judged by looking under the subject of computer arithmetic on the Amazon web site. Over 15,000 book titles are listed.

47. R. B. Laughlin and D. Pines, Proc. Nat'l Acad. Sci. 97, pp 28-31, (2000).

48. D. Meyer, Scale Decoherence in Inhomogenous Potentials, Archive preprint quant-ph/9805039 Private communication.

49. D. Meyer, Phys. Rev. Lett. 83, 3751-3754, (1999).

50. A. Peres, Finite Precision Measurements Nullifies Euclid's Postulates, Archive preprint quant-ph/0310035

51. K. Yosida Functional Analysis fifth Edition, No 123 in A Series of Comprehensive Studies in Mathematics, Springer Verlag, New york, 1978. 